\title{
Microscopic Theory of Spontaneous Decay in a Dielectric
}

\author{
Hao Fu, P. R. Berman \\ Michigan Center for Theoretical Physics, \\ FOCUS Center, and Physics Department, \\ University of Michigan, Ann Arbor, Michigan 48109-1040
}

(Dated: today; Received textdate; Revised textdate; Accepted textdate)

\begin{abstract}
The local field correction to the spontanous dacay rate of an impurity source atom imbedded in a disordered dielectric is calculated to second order in the dielectric density. The result is found to differ from predictions associated with both "virtual" and "real" cavity models of this decay process. However, if the contributions from two dielectric atoms at the same position are included, the virtual cavity result is reproduced.
\end{abstract}




\section{INTRODUCTION}

The problem of spontaneous emission from an atom imbedded inside a dielectric has attracted considerable interest [1]. Most theoretical treatments of this problem follow a macroscopic approach [2]. Based on different models of the local environment of the imbedded atom, they give different types of local field corrections to the spontaneous decay rate $\Gamma_{0}$ of the impurity atom. The so-called virtual cavity model gives a decay rate, $\Gamma_{\text {virtual }}=\sqrt{\epsilon}\left(\frac{\epsilon+2}{3}\right)^{2} \Gamma_{0}$, assuming that a virtual cavity surrounds the emitter, while the "real" cavity model gives a rate, $\Gamma_{\text {real }}=\sqrt{\epsilon}\left(\frac{3 \epsilon}{2 \epsilon+1}\right)^{2} \Gamma_{0}$, assuming that an empty spherical cavity surrounds the emitter. The quantity $\epsilon$ is the permittivity of the dielectric, which is connected to the microscopic polarizability $\alpha$ by the Lorentz-Lorenz relation $\epsilon=1+\frac{N \alpha}{1-\frac{1}{3} N \alpha}$, where $N$ is the dielectric density. Expansions for the decay rates in powers of $N \alpha$ yield $\Gamma_{\text {real }}=\left[1+\frac{7}{6} N \alpha+\frac{19}{72}(N \alpha)^{2}+O(N \alpha)^{3}\right] \Gamma_{0}$ and $\Gamma_{\text {virtual }}=\left[1+\frac{7}{6} N \alpha+\frac{17}{24}(N \alpha)^{2}+O(N \alpha)^{3}\right] \Gamma_{0}$. To first order in $N \alpha$, the real and virtual cavity models give identical results, but they differ in higher order. To determine the validity range of these macroscopic models, calculations using a somewhat more fundamental approach are needed. Several attempts at such microscopic models involve (i) a polariton approach for crystals [3], (ii) a Green's function approach for crystals 4] and disordered dielectrics [5], and (iii) an amplitude approach for disordered dielectrics [6] [7]. In the polariton method, the interaction between the vacuum radiation field and the crystal atoms is solved exactly; the eigenmodes of this system are the polaritons. The source atom then decays by radiating polaritons. This polariton calculation agrees with the virtual cavity result [3]. In the Green's function approach, the modification of the decay rate results from scattering of radiation emitted from the source atom by the dielectric, calculated to all orders in the dielectric density. This calculation reproduces the virtual cavity result with the source atom at an interstitial position and the real cavity result with source atom at a substitutional position in the crystal 4]. For disordered dielectrics, the Green's function method gives the virtual cavity result [5]. The amplitude method represents a direct calculation of the modification of the decay rate as a perturbation series in $N \alpha$ [6] [7]. To first order in $N \alpha$, the radiation emitted by the source atom is scattered back to the source atom by a single dielectric atom; the resultant decay rate agrees with the both virtual and real cavity models to first order in $N \alpha[\underline{6}]$.

In this paper, the amplitude method is extended to second order by including scattering 
events in which the radiation emitted by the source atom is scattered back to the source atom by a combined scattering from two dielectric atoms. It will be seen that the result differs from those of both the real and virtual cavity models; however, when contributions to the decay rate originating from scattering by two dielectric atoms located at the same physical point are included, the calculation reverts to the virtual cavity model.

\section{CALCULATION OF SECOND ORDER CONTRIBUTION}

The source atom located at $\mathbf{R}=0$, has a $J=0$ ground state and a $J=1$ excited state, the frequency separation of the ground and excited state denoted by $\omega_{0}$. The uniformly distributed dielectric atoms have $J=0$ ground states and $J=1$ excited states, the frequency separation of the ground and excited state denoted by $\omega$. At $t=0$, the source atom is excited to the $m=0$ excited state sublevel, the dielectric atoms are all in their ground states, and there are no photons in the field. The process we consider is radiation emitted by the source atom that is scattered by two dielectric atoms back to the source atom. It is assumed that $\left|\omega-\omega_{0}\right| / \Gamma_{0} \gg 1$ but that $\left|\omega-\omega_{0}\right| /\left(\omega+\omega_{0}\right) \ll 1$ [rotating wave approximation (RWA)].

We use a multipolar Hamiltonian [8]. The free part is

$$
H_{0}=\frac{\hbar \omega_{0}}{2} \sigma_{z}+\sum_{j} \sum_{m=-1}^{1} \frac{\hbar \omega}{2} \sigma_{z}^{(j)}(m)+\hbar \omega_{\mathbf{k}} a_{\mathbf{k} \lambda}^{\dagger} a_{\mathbf{k} \lambda},
$$

where $\sigma_{z}=(|2\rangle\langle 2|-| 1\rangle\langle 1|),|2\rangle$ and $|1\rangle$ are the $m=0$ excited and $J=0$ ground state eigenkets of the source atom, respectively, $\sigma_{z}^{(j)}(m)=\left(|m\rangle^{(j)}\langle m|-| g\rangle^{(j)}\langle g|\right)$ is the population difference operator between excited state $|J=1, m\rangle$ and ground state $|J=0, g\rangle$ of dielectric atom $j$, and $a_{\mathbf{k} \lambda}$ is the annihilation operator for a photon having momentum $\mathbf{k}$ and polarization $\lambda$. A summation convention is used, in which any repeated symbol on the right hand side of an equation is summed over, unless it also appears on the left-hand side of the equations.

The interaction part of the Hamiltonian is,

$$
V=-\mathbf{d}_{0} \cdot \frac{\mathbf{D}(0)}{\epsilon_{0}}-\mathbf{d}_{j} \cdot \frac{\mathbf{D}\left(\mathbf{R}_{j}\right)}{\epsilon_{0}},
$$

where $\mathbf{d}_{0}$ and $\mathbf{d}_{j}$ are the dipole operators of the source atom located at the origin and a dielectric atom located at position $\mathbf{R}_{j}$ respectively. The operator $\mathbf{D}$ is the displacement field 
having positive frequency component

$$
\mathbf{D}_{+}(\mathbf{R})=i \epsilon_{0} \sum_{\mathbf{k}, \lambda} \sqrt{\frac{\hbar \omega_{\mathbf{k}}}{2 \epsilon_{0} V}} \epsilon_{\mathbf{k}}^{(\lambda)} a_{\mathbf{k} \lambda} e^{i \mathbf{k} \cdot \mathbf{R}}
$$

where $V$ is the quantization volume and $\epsilon_{\mathbf{k}}^{(\lambda)}$ is a unit polarization vector, with

$$
\begin{aligned}
\epsilon_{\mathbf{k}}^{(1)} & =\cos \theta_{\mathbf{k}} \cos \phi_{\mathbf{k}} \hat{\mathbf{x}}+\cos \theta_{\mathbf{k}} \sin \phi_{\mathbf{k}} \hat{\mathbf{y}}-\sin \theta_{\mathbf{k}} \hat{\mathbf{z}} \\
\epsilon_{\mathbf{k}}^{(2)} & =-\sin \phi_{\mathbf{k}} \hat{\mathbf{x}}+\cos \phi_{\mathbf{k}} \hat{\mathbf{y}} .
\end{aligned}
$$

In the RWA, one can write

$$
\begin{gathered}
V=\sum_{\mathbf{k}} \hbar g_{\mathbf{k}}\left(\sigma_{+} a_{\mathbf{k}}-a_{\mathbf{k}}^{\dagger} \sigma_{-}\right)+\sum_{\mathbf{k}, \lambda, m} \hbar\left(g_{\mathbf{k} \lambda}^{\prime}(m) \sigma_{+}^{(j)}(m) a_{\mathbf{k} \lambda} e^{i \mathbf{k} \cdot \mathbf{R}}+g_{\mathbf{k} \lambda}^{\prime}(m)^{*} a_{\mathbf{k} \lambda}^{\dagger} \sigma_{-}^{(j)}(m) e^{-i \mathbf{k} \cdot \mathbf{R}}\right) \\
g_{\mathbf{k}}=-i \sqrt{\frac{\omega_{\mathbf{k}}}{2 \hbar \epsilon_{0} V}} \mu\left(\epsilon_{\mathbf{k}}^{(\lambda)}\right)_{0} \\
g_{\mathbf{k} \lambda}^{\prime}=-i \sqrt{\frac{\omega_{\mathbf{k}}}{2 \hbar \epsilon_{0} V}} \mu^{\prime}\left(\epsilon_{\mathbf{k}}^{(\lambda)}\right)_{m}^{*},
\end{gathered}
$$

where the $\sigma_{ \pm}$are raising and lowering operators for the source atom and $\sigma_{ \pm}^{(j)}(m)$ are raising and lowering operators between the excited state $|J=1, m\rangle$ and the ground state $|J=0, g\rangle$ of dielectric atom $j, \mu$ is the reduced matrix element of the dipole operator $\mathbf{d}_{0}$ and $\mu^{\prime}$ is that of $\mathbf{d}_{j}$ between ground and excited state manifolds. The $\left(\epsilon_{\mathbf{k}}^{(\lambda)}\right)_{ \pm 1}=\mp \frac{\left(\epsilon_{\mathbf{k}}^{(\lambda)}\right)_{x} \pm i\left(\epsilon_{\mathbf{k}}^{(\lambda)}\right)_{y}}{\sqrt{2}}$, $\left(\epsilon_{\mathbf{k}}^{(\lambda)}\right)_{0}=\left(\epsilon_{\mathbf{k}}^{(\lambda)}\right)_{z}$ are spherical components of the polarization vectors. The source atom interacts only with the $z$ component of the radiation field.

The calculation proceeds as in Ref. 6], with the addition of terms that couple dielectric atoms to dielectric atoms via the radiation field. After eliminating intermediate states involving the radiation field, one arrives at

$$
\begin{aligned}
\dot{b}_{2} & =-\gamma b_{2}-\gamma\left(\frac{\mu^{\prime}}{\mu}\right) e^{i \Delta t} G_{0, m_{j}}\left(\mathbf{R}_{j}, \omega\right) b_{m_{j}}(t) \\
\dot{b}_{m_{j}} & =-\gamma^{\prime} b_{m_{j}}-\gamma\left(\frac{\mu^{\prime}}{\mu}\right) e^{-i \Delta t} G_{m, 0}\left(\mathbf{R}_{j}, \omega_{0}\right) b_{2}(t)-\gamma^{\prime} G_{m_{j}, m_{s}^{\prime}}\left(\mathbf{R}_{j}-\mathbf{R}_{s}, \omega\right) b_{m_{s}^{\prime}}(t),
\end{aligned}
$$

where $\gamma=2 \mu^{2} \omega_{0}^{3} / 3 \hbar c^{3}$ and $\gamma^{\prime}=2 \mu^{\prime 2} \omega_{0}^{3} / 3 \hbar c^{3}$ are (half) the excited state decay rate of the source and dielectric atoms, respectively, $b_{2}$ is the state amplitude for the source atom to be in state $|2\rangle=|J=1, m=0\rangle$ and all dielectric atoms in their ground states, and $b_{m_{j}}$, is the state amplitude for dielectric atom $j$ to be in excited state $|J=1, m\rangle$ all other atoms in 
their ground states. We have set $b_{2}(t-\tau) \approx b_{2}(t)$ and $b_{m_{j}}(t-\tau) \approx b_{m_{j}}(t)$ on the assumption that $\gamma R_{0} / c, \gamma^{\prime} R_{0} / c \ll 1$, where $R_{0}$ is the sample size. The quantity $G_{m_{j}, m_{s}^{\prime}}\left(\mathbf{R}_{j}-\mathbf{R}_{s}, \omega\right)$ is a propagator for scattering from a dielectric atom in sublevel $m_{j}$ at position $\mathbf{R}_{j}$ to one in sublevel $m_{s}$ at position $\mathbf{R}_{s}$ given by

$$
G_{m_{j}, m_{s}^{\prime}}(\mathbf{R}, \omega)=\frac{3}{8 \pi} \frac{1}{\pi \omega^{3}} \int_{0}^{t} d \tau \int_{-\infty}^{\infty} d \omega_{k} \omega_{k}^{3} e^{-i\left(\omega_{k}-\omega\right) \tau} \int d \Omega_{\mathbf{k}}\left(\epsilon_{\mathbf{k}}^{(\lambda)}\right)_{m_{j}}^{*}\left(\epsilon_{\mathbf{k}}^{(\lambda)}\right)_{m_{s}^{\prime}} e^{i \mathbf{k} \cdot \mathbf{R}}
$$

while $G_{m, 0}\left(\mathbf{R}_{j}, \omega\right)$ is a propagator for scattering from the source atom to a dielectric atom in sublevel $m_{j}$ at position $\mathbf{R}_{j}$. In what follows we ignore the difference between $\omega_{0}$ and $\omega$, consistent with the RWA.

In order to solve Eqs. (9a 9b), we assume that $b_{2}$ varies slowly on the time scale $1 / \Delta$. If $b_{m_{j}}=y_{m_{j}} e^{-i \Delta t}$, Eqs. (9a 9b are transformed to

$$
\begin{gathered}
\dot{b}_{2}=-\gamma b_{2}-\gamma\left(\frac{\mu^{\prime}}{\mu}\right) G_{0, m}\left(\mathbf{R}_{j}, \omega_{0}\right) y_{m_{j}}(t) \\
\left(\gamma^{\prime}-i \Delta\right) y_{m_{j}}=-\gamma\left(\frac{\mu^{\prime}}{\mu}\right) G_{m, 0}\left(\mathbf{R}_{j}, \omega_{0}\right) b_{2}(t)-\gamma^{\prime} G_{m_{j}, m_{s}^{\prime}}\left(\mathbf{R}_{j}-\mathbf{R}_{s}, \omega_{0}\right) y_{m_{s}^{\prime}}(t)
\end{gathered}
$$

The formal solution for $\dot{b}_{2}$ is

$$
\dot{b}_{2}=-\gamma b_{2}+\gamma\left(\frac{\mu^{\prime}}{\mu}\right) G_{0, m_{j}}\left(\mathbf{R}_{j}, \omega_{0}\right)\left[\frac{1}{\gamma^{\prime}-i \Delta+\gamma^{\prime} \mathbf{G}}\right]_{m_{j}, m_{s}^{\prime}} \gamma\left(\frac{\mu^{\prime}}{\mu}\right) G_{m_{s}^{\prime}, 0}\left(\mathbf{R}_{s}, \omega_{0}\right) b_{2}
$$

where $\mathbf{G}$ is an $3 N \times 3 N$ matrix having matrix elements $G_{m_{j}, m_{s}^{\prime}}\left(\mathbf{R}_{j}-\mathbf{R}_{s}, \omega_{0}\right)$. This can be expanded as a power series in $N \alpha$ with $\alpha=-\frac{4 \pi \mu^{\prime 2}}{\hbar \Delta}$. To second order, one finds

$$
\dot{b}_{2,0}=-\gamma b_{2,0}\left[\begin{array}{c}
1+i N \alpha \frac{k_{0}^{3}}{6 \pi N} G_{0, m_{j}}\left(\mathbf{R}_{j}, \omega_{0}\right) G_{m_{j}, 0}\left(\mathbf{R}_{j}, \omega_{0}\right) \\
-(N \alpha)^{2}\left(\frac{k_{0}^{3}}{6 \pi N}\right)^{2} G_{0, m_{j}}\left(\mathbf{R}_{j}, \omega_{0}\right) G_{m_{j}, m_{s}^{\prime}}\left(\mathbf{R}_{j}-\mathbf{R}_{s}, \omega_{0}\right) G_{m_{s}^{\prime}, 0}\left(\mathbf{R}_{s}, \omega_{0}\right)
\end{array}\right]
$$

The term linear in the density gives the first order local field correction $\frac{\delta \gamma^{(1)}}{\gamma}=\frac{7}{6} N \alpha[6]$, which agrees with both the virtual and real cavity models to this order.

We now calculate the second order correction,

$$
\frac{\delta \gamma^{(2)}}{\gamma}=-(N \alpha)^{2}\left(\frac{k_{0}^{3}}{6 \pi N}\right)^{2} G_{0, m_{j}}\left(\mathbf{R}_{j}, \omega_{0}\right) G_{m_{j}, m_{s}^{\prime}}\left(\mathbf{R}_{j}-\mathbf{R}_{s}, \omega_{0}\right) G_{m_{s}^{\prime}, 0}\left(\mathbf{R}_{s}, \omega_{0}\right) .
$$

The sum over $\mathbf{R}_{j}$ and $\mathbf{R}_{s}$ can be converted to integrals using $\sum \rightarrow N \int d \mathbf{R}$. In this manner, one finds

$$
\frac{\delta \gamma^{(2)}}{\gamma}=-(N \alpha)^{2}\left(\frac{k_{0}^{3}}{6 \pi}\right)^{2} \iint d \mathbf{R}_{2} d \mathbf{R}_{1} G_{0, m_{j}}\left(\mathbf{R}_{2}, \omega_{0}\right) G_{m_{j}, m_{s}^{\prime}}\left(\mathbf{R}, \omega_{0}\right) G_{m_{s}^{\prime}, 0}\left(\mathbf{R}_{1}, \omega_{0}\right)
$$


where $\mathbf{R}=R_{2}-R_{1}$. The next step is to evaluate the $G_{m_{j}, m_{s}^{\prime}}(\mathbf{R}, \omega)$. The details of the calculation are given in the Appendix and one obtains

$$
\begin{aligned}
G_{11} & =\sqrt{4 \pi} h_{0}\left(k_{0} R\right) Y_{0,0}(\hat{\mathbf{R}})-\frac{1}{2} \sqrt{\frac{4 \pi}{5}} h_{2}\left(k_{0} R\right) Y_{2,0}(\hat{\mathbf{R}}) ; \\
G_{00} & =\sqrt{4 \pi} h_{0}\left(k_{0} R\right) Y_{0,0}(\hat{\mathbf{R}})+\sqrt{\frac{4 \pi}{5}} h_{2}\left(k_{0} R\right) Y_{2,0}(\hat{\mathbf{R}}) ; \\
G_{1,-1} & =-\frac{3}{2} \sqrt{\frac{8 \pi}{15}} h_{2}\left(k_{0} R\right) Y_{2,-2}(\hat{\mathbf{R}}) ; \\
G_{-1,1} & =-\frac{3}{2} \sqrt{\frac{8 \pi}{15}} h_{2}\left(k_{0} R\right) Y_{2,2}(\hat{\mathbf{R}}) ; \\
G_{1,0} & =-\frac{3}{2} \sqrt{\frac{4 \pi}{15}} h_{2}\left(k_{0} R\right) Y_{2,-1}(\hat{\mathbf{R}}) ; \\
G_{-1,0} & =-\frac{3}{2} \sqrt{\frac{4 \pi}{15}} h_{2}\left(k_{0} R\right) Y_{2,1}(\hat{\mathbf{R}}),
\end{aligned}
$$

where $Y_{\ell, m}(\hat{\mathbf{R}})$ is a spherical harmonic and $k_{0}=\omega_{0} / c$. The remaining $G_{m_{j}, m_{s}^{\prime}} \mathrm{s}$ are obtained using $G_{-1,-1}=G_{11}, G_{0,-1}=-G_{1,0}$, and $G_{0,1}=-G_{-1,0}$. The spherical Hankel functions of the first kind, $h_{0}\left(k_{0} R\right)$ and $h_{2}\left(k_{0} R\right)$, conform to the appropriate boundary conditions in which only outgoing scattered waves are considered.

The calculation for $\frac{\delta \gamma^{(2)}}{\gamma}$ is tedious, since it involves contributions from nine terms. We will show how to calculate one specific contribution, $m_{j}=1, m_{s}^{\prime}=1$, and then give the final results for the other components. Substituting Eqs. (16a 16e) in Eq. (15), we find

$$
\begin{aligned}
\frac{\delta \gamma^{(2)}(1,1)}{\gamma} & =(N \alpha)^{2}\left(\frac{k_{0}^{3}}{6 \pi}\right)^{2} \frac{6 \pi^{\frac{3}{2}}}{5} \iint d \mathbf{R}_{1} d \mathbf{R}_{2} h_{2}\left(k_{0} R_{2}\right) Y_{2,1}\left(\hat{\mathbf{R}}_{2}\right) h_{0}\left(k_{0} R_{21}\right) Y_{0,0}\left(\hat{\mathbf{R}}_{21}\right) h_{2}\left(k_{0} R_{1}\right) Y_{2,-1}\left(\hat{\mathbf{R}}_{1}\right) \\
& -(N \alpha)^{2}\left(\frac{k_{0}^{3}}{6 \pi}\right)^{2} \frac{3 \pi^{\frac{3}{2}}}{5 \sqrt{5}} \iint d \mathbf{R}_{1} d \mathbf{R}_{2} h_{2}\left(k_{0} R_{2}\right) Y_{2,1}\left(\hat{\mathbf{R}}_{2}\right) h_{2}\left(k_{0} R_{21}\right) Y_{2,0}\left(\hat{\mathbf{R}}_{21}\right) h_{2}\left(k_{0} R_{1}\right) Y_{2,-1}\left(\hat{\mathbf{R}}_{1}\right) .
\end{aligned}
$$

To evaluate this, we expand the Hankel functions as [9]

$$
\begin{aligned}
h_{l}\left(k_{0} R_{21}\right) Y_{l, m}\left(\hat{\mathbf{R}}_{21}\right) & =i^{l_{1}+l_{2}-l}(-1)^{l_{2}+m} \sqrt{4 \pi(2 l+1)\left(2 l_{1}+1\right)\left(2 l_{2}+1\right)} \\
& \times\left[\Theta\left(R_{2}-R_{1}\right)+(-1)^{l} \Theta\left(R_{1}-R_{2}\right)\right]\left(\begin{array}{ccc}
l_{1} & l & l_{2} \\
0 & 0 & 0
\end{array}\right)\left(\begin{array}{ccc}
l_{1} & l & l_{2} \\
m_{1} & m & m_{2}
\end{array}\right) \\
& \times h_{l_{1}}\left(k_{0} R_{>}\right) j_{l_{2}}\left(k_{0} R_{<}\right) Y_{\ell_{1}, m_{1}}\left(\hat{\mathbf{R}}_{>}\right) Y_{\ell_{2}, m_{2}}\left(\hat{\mathbf{R}}_{<}\right)
\end{aligned}
$$

where $(\cdots)$ is a $3-j$ symbol, $j_{l}(x)$ is a spherical Bessel function, and $R_{>}\left(R_{<}\right)$is the larger (smaller) of $R_{1}$ and $R_{2}$. When this expansion is used in Eq. (17), the angular integration 
selects out only $l=2,0$ and $m=1,-1,0$ terms, such that

$$
\frac{\delta \gamma^{(2)}(1,1)}{\gamma}=-\frac{(N \alpha)^{2}}{7} \int_{0}^{\infty} d \rho_{2} \rho_{2}^{2} \int_{0}^{\rho_{2}} d \rho_{1} \rho_{1}^{2} h_{2}\left(\rho_{2}\right) h_{2}\left(\rho_{1}\right) h_{2}\left(\rho_{2}\right) j_{2}\left(\rho_{1}\right)
$$

with $\rho_{1}=k_{0} R_{1}, \rho_{2}=k_{0} R_{2}$. To evaluate the above integral, we add a convergence factor $e^{-\epsilon \rho_{2}}$, and eventually take the limit $\epsilon \rightarrow 0$. The imaginary part of the integral diverges as $\rho_{2} \rightarrow 0$, but the real part is finite and gives the local field correction to the decay rate. The result is

$$
\frac{\delta \gamma^{(2)}(1,1)}{\gamma}=\frac{15}{112}(N \alpha)^{2}
$$

and the corresponding results for the other terms are [10]

$$
\begin{aligned}
\frac{\delta \gamma^{(2)}(-1,-1)}{\gamma} & =\frac{\delta \gamma^{(2)}(1,1)}{\gamma}=\frac{15}{112}(N \alpha)^{2}, \\
\frac{\delta \gamma^{(2)}(0,0)}{\gamma} & =\frac{25}{63}(N \alpha)^{2}, \\
\frac{\delta \gamma^{(2)}(0,1)}{\gamma} & =\frac{\delta \gamma^{(2)}(1,0)}{\gamma}=\frac{\delta \gamma^{(2)}(-1,0)}{\gamma}=\frac{\delta \gamma^{(2)}(0,-1)}{\gamma}=\frac{3}{28}(N \alpha)^{2}, \\
\frac{\delta \gamma^{(2)}(-1,1)}{\gamma} & =\frac{\delta \gamma^{(2)}(1,-1)}{\gamma}=-\frac{3}{56}(N \alpha)^{2} .
\end{aligned}
$$

The total second order correction to the decay rate is

$$
\frac{\delta \gamma^{(2)}}{\gamma}=2 \frac{\delta \gamma^{(2)}(1,1)}{\gamma}+\frac{\delta \gamma^{(2)}(0,0)}{\gamma}+4 \frac{\delta \gamma^{(2)}(0,1)}{\gamma}+2 \frac{\delta \gamma^{(2)}(-1,1)}{\gamma}=\frac{71}{72}(N \alpha)^{2}
$$

This result differs from both the virtual $\left[\frac{51}{72}(N \alpha)^{2}\right]$ and real $\left[\frac{19}{72}(N \alpha)^{2}\right]$ cavity models .

Our result can be compared with Fleischhauer's [5]. The Fourier transform of $G_{m, m^{\prime}}\left(\mathbf{R}, \omega_{0}\right)$ is the tensor field propagator $\mathbf{F}^{(0)}(\mathbf{q}, \omega)$ in his paper, differing only by prefactors. The integral (15) can then be done in either coordinate or momentum space. The momentum space integration gives a different result than our coordinate space calculation above. This surprising discrepancy can be explained by the way we expand $h_{l}\left(k_{0} R_{21}\right) Y_{l, m}\left(\hat{\mathbf{R}}_{21}\right)$. The expansion we used is valid for $R_{1}>R_{2}$ or $R_{2}>R_{1}$, but is not defined for $R_{1}=R_{2}$. For a well-behaved integral this will not make any difference since $R_{1}=R_{2}$ contributes a set of measure zero. In the present case, however, where the dipoledipole interaction between dielectric atoms diverges when one atom is on the top the other, i.e. when $\mathbf{R}_{1}=\mathbf{R}_{2}$, the contribution from $R_{1}=R_{2}$ can be finite.

It is not easy to estimate this contribution in the original form of the integral (17). Instead, it proves useful to Fourier transform just one of the $G_{m, m^{\prime}}$ in the integrand. As an 
example, we consider the integral in the second term of Eq. (17)

$$
I=\iint d \mathbf{R}_{2} d \mathbf{R}_{1} h_{2}\left(k R_{2}\right) Y_{2,1}\left(\hat{\mathbf{R}}_{2}\right) h_{2}\left(k R_{21}\right) Y_{2,0}\left(\hat{\mathbf{R}}_{21}\right) h_{2}\left(k R_{1}\right) Y_{2,-1}\left(\hat{\mathbf{R}}_{1}\right)
$$

We Fourier transform $h_{2}\left(k R_{21}\right) Y_{2,0}\left(\hat{\mathbf{R}}_{21}\right) e^{-\epsilon R_{21}}$, using a convergence factor $e^{-\epsilon R_{21}}$ that is physically connected with the boundary condition of outgoing spherical waves. Carrying out the Fourier transform in Eq. (21), we find

$$
I=-\frac{4 \pi i}{k^{3}} \iint d \mathbf{R}_{2} d \mathbf{R}_{1} h_{2}\left(k R_{2}\right) Y_{2,1}\left(\hat{\mathbf{R}}_{2}\right) h_{2}\left(k R_{1}\right) Y_{2,-1}\left(\hat{\mathbf{R}}_{1}\right) \int \frac{d \mathbf{p}}{(2 \pi)^{3}} \frac{p^{2}}{k^{2}-p^{2}+i \epsilon} Y_{2,0}(\hat{\mathbf{p}}) e^{i \mathbf{p} \cdot\left(\mathbf{R}_{2}-\mathbf{R}_{1}\right)}
$$

The angular integrations can be done by expanding $e^{i \mathbf{p} \cdot \mathbf{R}_{2}}, e^{-i \mathbf{p} \cdot \mathbf{R}_{1}}$ in terms of spherical harmonics and Bessel functions. In this manner one obtains

$$
I=\frac{1}{14} \sqrt{\frac{5}{\pi}} \frac{(4 \pi)^{3} i}{k^{3}} \iint d R_{2} d R_{1} R_{2}^{2} R_{1}^{2} h_{2}\left(k R_{2}\right) h_{2}\left(k R_{1}\right) \int \frac{d p}{(2 \pi)^{3}} \frac{p^{4}}{k^{2}-p^{2}+i \epsilon}
$$

We are interested only in the contribution in the region where $R_{1}=R_{2}$. This contribution can be isolated by integrating $R_{2}$ from $R_{1}-a$ to $R_{1}+a$, and then integrating the resultant expression over $p$ using the method of residues. In the limit that both $a$ and $\epsilon$ tend to zero, one obtains the contribution $\delta I$ from the region $R_{1}=R_{2}$ as

$$
\operatorname{Re}[\delta I]=\operatorname{Re}\left[\frac{-1}{7} \sqrt{5 \pi} \frac{2 i}{k^{3}} \int d R_{2} R_{2}^{2} h_{2}\left(k R_{2}\right) h_{2}\left(k R_{2}\right)\right]=\frac{-5 \sqrt{5 \pi}}{7 k^{6}}
$$

(the imaginary part of $\delta I$ diverges). The contribution from the sphere $R_{1}=R_{2}$ is identical to that from $\mathbf{R}_{1}=\mathbf{R}_{2}$ since all other points with $\mathbf{R}_{1} \neq \mathbf{R}_{2}$ on the sphere are regular and contribute zero to the integral. The same calculation can be done for the first integral in the Eq. (17). For this term, there is no contribution from the region $R_{1}=R_{2}$ (no delta function like term is found) since $h_{0}\left(k R_{21}\right)$ has a lower order divergence at $R_{21}=0$ than does $h_{2}\left(k R_{21}\right)$.

Including contributions of the type (24), we find

$$
\begin{aligned}
\frac{\delta \gamma^{(2)}(0,0)}{\gamma} & =\frac{1}{3}(N \alpha)^{2} \\
\frac{\delta \gamma^{(2)}(1,1)}{\gamma} & =\frac{\delta \gamma^{(2)}(1,-1)}{\gamma}=\frac{7}{48}(N \alpha)^{2} \\
\frac{\delta \gamma^{(2)}(0,1)}{\gamma} & =\frac{\delta \gamma^{(2)}(1,0)}{\gamma}=\frac{\delta \gamma^{(2)}(-1,0)}{\gamma}=\frac{\delta \gamma^{(2)}(0,-1)}{\gamma}=\frac{1}{12}(N \alpha)^{2}, \\
\frac{\delta \gamma^{(2)}(-1,1)}{\gamma} & =\frac{\delta \gamma^{(2)}(1,-1)}{\gamma}=-\frac{1}{8}(N \alpha)^{2} .
\end{aligned}
$$

When these are summed the total $\frac{\delta \gamma^{(2)}}{\gamma}=\frac{17}{24}(N \alpha)^{2}$ agrees with the virtual cavity result. 


\section{DISCUSSION}

The second order contribution to the modified spontaneous emission rate of an impurity atom in a disordered dielectric has been calculated using a microscopic theory. Depending on the manner in which overlapping atoms are treated, one arrives at different results. If the delta function contributions at $\mathbf{R}_{1}=\mathbf{R}_{2}$ are included, the virtual cavity model is recovered, but if such terms are excluded, neither the real nor virtual cavity model results are found. It seems to us somewhat of an open question at this point as to whether or not such contributions can be uniquely calculated once Eq. (12) is expanded in a power series in the density. The reason for this is that the expansion parameter is not small as interatomic distances tend to zero. That the expansion can lead to divergences is already evident if the integrations are carried out using a different set of variables [10]. From physical considerations, however, the decay rate does not diverge, even for interparticle spacings much less than a wavelength. Actually, dielectric atoms within a sphere of radius $\lambda\left(\gamma^{\prime} / \Delta\right)^{1 / 3}$ reradiate collectively; outside this radius, there is destructive interference resulting in some additional finite contribution to the decay rate. In dealing with a homogeneous dielectric, we have performed the ensemble average by integrating over all space assuming a constant density. This averaging process includes configurations where interparticle spacings are sufficiently small to invalidate the expansion (13). Nevertheless, the procedure has yielded finite results for the change in the decay parameter.

Different experiments support both the real and virtual cavity results 11]. The source atom in these experiments is usually an impurity ion in a protective molecular cage. No experiments of this nature have been carried out with impurity atomic radiators in a dielectric that consists of a dense atomic vapor. It may be possible to use an alkali metal atom as the source atom and rare gas atoms as the dielectric atoms. With such a system, one could not make the rotating wave approximation used in this paper, but the physics is not changed in any substantive manner. The key feature of the alkali metal - rare gas system is the extremely small quenching cross sections for rare gas collisions to inelastically change

the electronic state of the alkali atom [12]. Any quenching cross sections would appear as a modification of the decay rate that would mask the sought after effect. For rare gas pressures on the order of 100 atmospheres, we estimate that a change in the decay rate of order of $3 \%$ could be observed. To increase the effect it is necessary to find radiator atoms whose first 
excited state is radiatively coupled to the ground state and dielectric atoms whose lowest excited state is about $0.2 \mathrm{eV}$ above the energy of the excited state of the radiator. In this limit, quenching will be negligible, but the detuning $\Delta$ is decreased from the alkali-rare gas system by a factor of 50. At the same time, it is necessary to achieve a high pressure for the dielectric atoms. A possible system would be Li radiators with a high density sodium dielectric; the energy mismatch of $\mathrm{Li}$ and $\mathrm{Na}$ is about $0.25 \mathrm{eV}$, giving a correction factor to the lithium decay rate of $1.3 \times 10^{-21} N$, where $N$ is the sodium dielectric density in units of atoms $/ \mathrm{cm}^{3}$.

\section{ACKNOWLEDGMENTS}

This research is supported by the National Science Foundation under Grants No. PHY0244841 and the FOCUS Center Grant. PRB would like to thank Peter Milonni for helpful comments and Georg Raithel for a discussion concerning high pressure sodium lamps.

\section{APPENDIX}

In this appendix, we calculate explicitly $G_{1,1}(\mathbf{R}, \omega)$ given in Eq. (10). The other $G_{m, m^{\prime}}(\mathbf{R}, \omega)$ are calculated in a similar fashion. To carry out the angular integrations, one expands $e^{i \mathbf{k} \cdot \mathbf{R}}$ as

$$
e^{i \mathbf{k} \cdot \mathbf{R}}=4 \pi \sum_{m=-l}^{l} i^{l} Y_{l m}^{*}(\hat{\mathbf{k}}) Y_{l m}(\hat{\mathbf{R}}) j_{l}(k R),
$$

uses the fact that $\left(\epsilon_{\mathbf{k}}^{(\lambda)}\right)_{1}^{*}\left(\epsilon_{\mathbf{k}}^{(\lambda)}\right)_{1}=\frac{1}{2}\left(1+\cos ^{2} \theta\right)=\frac{\sqrt{4 \pi}}{3}\left[2 Y_{00}(\hat{\mathbf{k}})+\frac{1}{\sqrt{5}} Y_{20}(\hat{\mathbf{k}})\right]$, and the orthogonality of the spherical harmonics, to obtain

$$
G_{1,1}(\mathbf{R}, \omega)=\frac{1}{\sqrt{\pi} \omega^{3}} \int_{0}^{t} d \tau \int_{-\infty}^{\infty} d \omega_{k} \omega_{k}^{3} e^{-i\left(\omega_{k}-\omega\right) \tau}\left[2 Y_{00}(\hat{\mathbf{R}}) j_{0}(k R)-\frac{1}{\sqrt{5}} Y_{20}(\hat{\mathbf{R}}) j_{2}(k R)\right]
$$

The spherical Bessel function can written in terms of spherical Hankel functions as $j_{l}(k R)=$ $\frac{1}{2}\left[h_{l}(k R)+h_{l}^{*}(k R)\right]$, transforming Eq. (27) into

$G_{1,1}(\mathbf{R}, \omega)=\frac{1}{2 \sqrt{\pi}} \int_{0}^{t} d \tau \int_{-\infty}^{\infty} d \omega_{k} e^{-i\left(\omega_{k}-\omega\right) \tau}\left\{2 Y_{00}(\hat{\mathbf{R}})\left[h_{0}(k R)+h_{0}^{*}(k R)\right]-\frac{1}{\sqrt{5}} Y_{20}(\hat{\mathbf{R}})\left[h_{2}(k R)+h_{2}^{*}(k R)\right]\right\}$.

In the calculation we always make the Wigner-Weisskopf approximation. Differences between $\omega, \omega_{0}$ and $\omega_{k}$ are neglected except they appear as exponential factors. In integrating 
over $\omega_{k}$, the $h_{l}^{*}(k R)$ terms give a contribution proportional to $\delta(R / c+\tau)$ while the $h_{l}(k R)$ terms give a contribution proportional to $\delta(R / c-\tau)$. We retain only the $\delta(R / c-\tau)$ contributions since they correspond to the retarded solution (outgoing spherical waves). As a consequence, we find

$$
G_{1,1}(\mathbf{R}, \omega)=\sqrt{4 \pi} h_{0}\left(k_{0} R\right) Y_{0,0}(\hat{\mathbf{R}})-\frac{1}{2} \sqrt{\frac{4 \pi}{5}} h_{2}\left(k_{0} R\right) Y_{2,0}(\hat{\mathbf{R}})
$$

[1] D. Toptygin, J. Fluorensc. 13, 201 (2003); A. Luks and V. Perinova, Progress in Opitcs, edited by E. Wolf (Elsevier, Amsterdam, 2002), vol. 43, pp. 295-431; A. Thranhardt, C. Ell, G. Khitrova, and H. M. Gibbs, Phys, Rev. B 65, 035327 (2002), and references therein.

[2] S. Scheel, L. Knoll, D.-G. Welsch, and S.M. Barnett, Phys. Rev. A 60, 1590 (1999), and references therein

[3] J. Knoester and S. Mukamel, Phys. Rev. A 40, 7065

[4] Pedro de Vries and Ad Lagendijk, Phys. Rev. Let. 81, 1381 (1998) and reference therein

[5] M. Fleischhauer Phys. Rev. A 60, 2534 (1999) and reference therein

[6] P. R. Berman and P. W. Milonni Phys. Rew. Lett. 92, 053601 (2004)

[7] M. E. Crenshaw and C. M. Bowden, Phys. Rew. Lett. 85, 1851 (2000)

[8] E. A. Power Introductory Quantum Electrodynamics (Elsevier, New York, 1965) Chapter 8

[9] M. Danos and L. C. Maximon J. of Math. Phys. 6, 766 (1965)

[10] The contributions from other terms may involve factors such as $h_{0}\left(k_{0} R_{1}\right) h_{2}\left(k_{0} R_{21}\right) h_{2}\left(k_{0} R_{2}\right)$. Direct expansion of $h_{2}\left(k_{0} R_{21}\right)$ gives a divergent result for the decay rate, when the contribution from $R_{1}=R_{2}$ is excluded. Instead, we expand $h_{0}\left(k_{0} R_{1}\right)$ in terms of $R_{21}$ and $R_{2}$ and integrate over these two variables. This procedure leads to the finite results given in the paper. The fact that we get divergent results when directly expanding $h_{2}\left(k_{0} R_{21}\right)$ in terms of $R_{1}$ and $R_{2}$ can be traced to the fact that one finds different values for the integrals at $R_{1}=R_{2}$ depending on whether the limit $R_{1}=R_{2}$ is approached from above or below. If the contribution from $R_{1}=R_{2}$ is included, it leads to a divergence that exactly cancels the divergence from the first integral and again leads to the same result given in the text.

[11] Different experiments have lent support to the virtual-cavity formula [see, for instance, R. S. Meltzer, S. P. Feofilov, B. Tissue, and H. B. Yuan, Phys. Rev. B 60, 14012 (1999)] as well as 
the real-cavity formula [see, for instance, G. L. J. A. Rikken and Y. A. R. R. Kessener, Phys. Rev. Lett. 74, 880 (1995) or F. J. P. Schuurmans, D. T. N. de Lang, G. H. Wegdam, R. Sprik, and A. Lagendijk, Phys. Rev. Lett. 80, 5077 (1998)]

[12] See, for example, J. H. Stamper, Phys. Rev. J. Chem Phys. 43, 759 (1965); M. G. Edwards, J. Phys. B 2, 719 (1969); E. Speller, B. Staudenmayer, and V. Kempter, Z. Phys. A 291, 311 (1979). 